\title{
ADDENDUM: STABLE AND REGULAR REACHABILITY OF RELAXED HEREDITARY DIFFERENTIAL SYSTEMS*
}

\author{
FRITZ COLONIUS $\dagger$
}

\begin{abstract}
This paper characterizes regular reachability of relaxed hereditary differential systems as a positive controllability property of an associated linear system in the Sobolev space $W^{\mathbf{1}, 2}$. Thus the results of F. Colonius [SIAM J. Control Optim., 20 (1982), pp. 675-694] are improved. Regular reachability is relevant as a regularity condition in the proof of a maximum principle for fixed final state optimal control problems.
\end{abstract}

The paper [2] considered optimal control problems for relaxed hereditary differential systems of the form

$$
x_{t_{0}}=\phi^{0}, \quad \dot{x}(t)=f\left(x_{t}, v(t), t\right) \quad \text { a.a. } t \in T:=\left[t_{0}, t_{1}\right] .
$$

For problems with a fixed final state $x_{t_{1}}=\phi \in W^{1,2}\left([-r, 0], \mathbb{R}^{n}\right)$, a maximum principle was proved, provided that the optimal solution $\left(x^{0}, v^{0}\right)$ satisfies the following regularity condition for some $\delta>0$ :

$$
\dot{\phi}\left(t-t_{1}\right) \in \operatorname{int}_{\delta} \operatorname{co} f\left(x_{t}^{0}, \Omega(t), t\right) \quad \text { a.a. } t \in T_{1}:=\left[t_{1}-r, t_{1}\right]
$$

(the abbreviations $T$ and $T_{1}$ introduced above will be used throughout this note).

The paper [3] was devoted to the problem of understanding the regularity condition (2). However, no complete characterization in terms of controllability properties of a linearized system could be given. The present note solves this problem. This gives deeper insight into the relations between optimal control and structure theory of hereditary differential systems and provides the missing link between the special situation of [2] and the general Banach space setting of [4].

In the following, we assume that $\phi$ is continuously differentiable and that $f$ and the set $\Omega$ do not depend on time $t$. This is in order not to overburden this note with technical details.

Furthermore, we suppose that the assumptions (1.1), (1.2), and (1.4) of [3] are satisfied, and define for an interval $I \subset T$ and $p=2$ or $p=\infty$

$$
U_{p}(I):=\left\{u \in L_{p}\left(I ; \mathbb{R}^{n}\right): u(t) \in P(t):=\mathbb{R}_{+}\left(\operatorname{co} f\left(x_{t}^{0}, \Omega\right)-f\left(x_{t}^{0}, v^{0}(t)\right) \text { for a.a. } t \in I\right\} ;\right.
$$

here $\mathbb{R}_{+}$is the set of all nonnegative reals.

Along with (1) we consider the following linearized system $(p=2$ or $p=\infty)$ :

$$
\begin{aligned}
& x_{t_{0}}=0, \quad \dot{x}(t)=\mathscr{D}_{1} f\left(x_{t}^{0}, v^{0}(t)\right) x_{t}+u(t) \quad \text { a.a. } t \in T, \\
& u \in \mathscr{U}_{p}(T) .
\end{aligned}
$$

The present analysis differs in two essential points from the previous one in [3]. (a) We consider the final states $x_{t_{1}}$ of the linearized system in $W^{1,2}\left([-r, 0], \mathbb{R}^{n}\right)$, instead of $W^{1, \infty}\left([-r, 0], \mathbb{R}^{n}\right)$. (b) In [3, Lemma 1.5], the control values $u(t)$ of the linearized system were required to lie in $\operatorname{co} f\left(x_{t}^{0}, \Omega\right)-f\left(x_{t}^{0}, v^{0}(t)\right)$, while (4) above allows the control values to lie in the closed convex cone (with vertex at 0 ) generated by this set. Thus the admittance of $L_{2}$-controls instead of $L_{\infty}$-controls significantly changes the situation as we will see in a moment.

* This Journal, 20 (1982), pp. 675-694. Addendum received by the editors March 12, 1984, and in revised form October $15,1984$.

$\dagger$ Division of Applied Mathematics, Brown University, Providence, Rhode Island 02912. Part of this research was performed during a visit to the Institut für Mathematik der Universität Graz supported by Deutsche Forschungsgemeinschaft. 
THEOREM 1. The following two conditions are equivalent:

(i) $\left\{(\dot{x})_{t_{1}}: x\right.$ solves $(3)$ for some $\left.u \in \mathcal{U}_{2}(T)\right\}=L_{2}\left([-r, 0], \mathbb{R}^{n}\right)$.

(ii) $P(t)=\mathbb{R}^{n}$ for a.a. $t \in T_{1}$.

Furthermore if for a.a. $t \in T_{1}$ the cone $P(t)$ is a subspace of $\mathbb{R}^{n}$, the following two conditions are equivalent: a.e.\}.

(iii) $\mathcal{U}_{\infty}\left(T_{1}\right)=\left\{\lambda u: \lambda \in \mathbb{R}_{+}, u \in L_{\infty}\left(T_{1}, \mathbb{R}^{m}\right)\right.$ with $u(t) \in \operatorname{co} f\left(x_{t}^{0}, \Omega\right)-f\left(x_{t}^{0}, v^{0}(t)\right)$

(iv) For some $\delta>0$

$$
0 \in \operatorname{relint}_{\delta} \operatorname{co} f\left(x_{t}^{0}, \Omega\right)-f\left(x_{t}^{0}, v^{0}(t)\right) \quad \text { for a.a. } t \in T_{1} \text {. }
$$

Here relint $Q$ of $Q \subset \mathbb{B}^{n}$ denotes the interior of $Q$ with respect to the smallest linear subspace containing $Q$.

The proof of these results will be postponed until after Theorem 2. First we discuss their significance.

It is immediate from the proof of Theorem 1 that the set at the left-hand side of the equation in (i) does not change, if $u \mid\left[t_{0}, t_{1}-t\right]$ is required to lie in $L_{\infty}$. The cone $\left\{\lambda u: \lambda \in \mathbb{R}_{+}, u \in L_{\infty}\left(T, \mathbb{R}^{m}\right)\right.$ with $u(t) \in \operatorname{co} f\left(x_{t}^{0}, \Omega\right)-f\left(x_{t}^{0}, v^{0}(t)\right)$ a.e. $\}$ corresponds to the cone of admissible directions for the control constraint in $L_{\infty}$ in the fixed final state optimal control problem. By [4, Example 1.1], the $L_{2}$-closure of $U_{\infty}\left(T_{1}\right)$ coincides with $\mathcal{U}_{2}\left(T_{1}\right)$. Hence, taken together, the regularity condition (2) (being equivalent to (ii), (iv)) means by (i) and (iii) that the $L_{2}$-closure of the cone of admissible directions in $L_{\infty}$ is mapped onto $L_{2}\left([-r, 0], \mathbb{R}^{n}\right)$ under the linearized control-to-final-state-velocitymap. Thus the assumptions of [4, Thm. 1.2] can be verified and Lagrange multipliers in $W^{1, \infty}\left([-r, 0], \mathbb{R}^{n}\right)$ can be identified with functions in $W^{1,2}\left([-r, 0], \mathbb{R}^{n}\right)$ (observe that the finite dimensional part $x\left(t_{1}-r\right)=\phi(-r)$ does not pose any problem here).

Furthermore, Theorem 1 shows very clearly, where the uniformity condition (that is the $\delta$-bound) in (2) comes in: It guarantees that the cone $U_{\infty}$ defined by pointwise restrictions is not bigger than the cone of admissible directions (on the relevant interval $\left.T_{1}\right)$.

Thus Theorem 1 clarifies the relation between the regularity condition (2) and the required controllability condition for a linearized system and embeds the special situation of [2] into the general Banach space setting of [4].

Remark 1. The role of the uniformity condition as interpreted above shows that the regularity condition might be weakened somewhat, since we are only interested in the $L_{2}$-closure of the cone of admissible directions: If co $f\left(x_{t}^{0}, \Omega\right)-f\left(x_{t}^{0}, v^{0}(t)\right)$ "shrinks fast enough" around zero for some point $\bar{t} \in T_{1}$ and (ii) is satisfied, $U_{2}\left(T_{1}\right)$ will still coincide with this $L_{2}$-closure and the surjectivity condition is satisfied.

For a proof of the result above, we consider the following slightly more general problem of controllability under positivity constraints for the control:

$$
\begin{array}{ll}
x_{t_{0}}=0, \quad \dot{x}(t)=L(t) x_{t}+B(t) u(t) & \text { a.a. } t \in T, \\
u(t) \in P(t) & \text { a.a. } t \in T
\end{array}
$$

where $\quad L: T \rightarrow \mathscr{L}\left(C\left([-r, 0], \mathbb{R}^{n}\right), \mathbb{R}^{n}\right) \quad$ with $\quad t \mapsto L(t) \phi$ measurable for all $\phi \in$ $C\left([-r, 0], \mathbb{R}^{n}\right)$ and ess sup $\|L(t)\|<\infty, B \in L_{\infty}\left(T, \mathbb{R}^{n \times m}\right) ;$ and $P(t) \subset \mathbb{R}^{m}$ is a closed convex cone with vertex at zero and $t \mapsto P(t)$ measurable (see $[1$, p. 68]) (L्L denotes the space of bounded linear maps). 
We say that $(5),(6)$ is completely controllable to $W^{1,2}\left([-r, 0], \mathbb{R}^{n}\right)$ if the reachable set $\mathscr{R}$ defined by

$$
\mathscr{R}:=\left\{x_{t_{1}}: x \text { solves }(5) \text { for some control } u \in L_{2}\left(T, \mathbb{R}^{m}\right) \text { satisfying (6) }\right\},
$$

coincides with this space.

Define the multiplication operator $B: L_{2}\left(T_{1}, \mathbb{R}^{m}\right) \rightarrow L_{2}\left(T_{1}, \mathbb{R}^{n}\right)$ by

$$
(\tilde{B} u)(t):=B(t) u(t) \quad \text { a.a. } t \in T_{1},
$$

and define the closed convex cone $\tilde{P} \subset L_{2}\left(T_{1}, \mathbb{R}^{m}\right)$ by

$$
\tilde{P}:=\left\{u \in L_{2}\left(T_{1}, \mathbb{R}^{m}\right): u(t) \in P(t) \text { a.e. }\right\} .
$$

LEMMA. Suppose that the generalized inverse $B(t)^{+}$of $B(t)$ has essentially bounded norm on $T_{1}$. Then $\tilde{B} \tilde{P}=L_{2}\left(T_{1}, \mathbb{R}^{n}\right)$ iff $B(t) P(t)=\mathbb{R}^{n}$ a.e. on $T_{1}$.

Proof. One direction is trivial. Conversely, suppose that $B(t) P(t)=\mathbb{R}^{n}$ a.e. on $T_{1}$. By [5, Lemma 3] our hypothesis means that $\tilde{B}$ has a closed range. Consider the set

$$
\left\{u \in L_{2}\left(T_{1}, \mathbb{R}^{m}\right): u(t) \in P_{1}(t) \text { a.e. }\right\}
$$

where $P_{1}(t)$ is the projection of $P(t)$ to $[\operatorname{Ker} B(t)]^{\perp}$. This set is a closed linear subspace which is mapped onto a closed linear subspace $X$ of $L_{2}\left(T_{1}, \mathbb{R}^{n}\right)$, since $\tilde{B} \mid\{u \in$ $L_{2}\left(T_{1}, \mathbb{R}^{m}\right): u(t) \in[\operatorname{ker} B(t)]^{\perp}$ a.e. $\}$ is a homeomorphism onto the image of $\tilde{B}$. Thus the space $X=\tilde{B} \tilde{P}$ is a closed linear subspace of $L_{2}$ which naturally is also dense. This proves the assertion.

We obtain the following result.

THEOREM 2. Suppose that the generalized inverse $B(t)^{+}$of $B(t)$ has an essentially bounded norm on $T_{1}$. Then system $(5),(6)$ is completely controllable to $W^{1,2}\left([-r, 0], \mathbb{R}^{n}\right)$ iff the following two conditions are satisfied:

(i) $\mathscr{R}^{f}:=\left\{x\left(t_{1}-r\right): x\right.$ solves (5) for some control $\left.u \in \tilde{P}\right\}=\mathbb{R}^{n}$;

(ii) $B(t) P(t)=\mathbb{R}^{n}$ a.e. on $T_{1}$.

Proof. By the lemma above conditions (i) and (ii) imply $\mathscr{R}=W^{1,2}\left([-r, 0], \mathbb{R}^{n}\right)$. Conversely, condition (i) follows trivially. Suppose that condition (ii) is violated, i.e. there is a subset $T_{2} \subset T_{1}$ of positive Lebesgue measure such that

$$
0 \in \partial B(t) P(t), \quad t \in T_{2} ;
$$

here $\partial$ denotes the boundary.

Equivalently,

$$
0 \in \partial\{B(t) P(t) \cap \mathscr{E}\}
$$

where $\mathscr{E}:=\left\{y \in \mathbb{R}^{n}:|y|=1\right\}$.

Define for $t \in T_{2}$

$$
\Gamma(t):=\left\{y \in \mathbb{R}^{n}: y \in \mathscr{E} \text { and }\langle y, B(t) p\rangle \leqq 0 \text { for all } p \in P(t)\right\},
$$

Then $\Gamma$ has compact values and is measurable. Hence there is a measurable selection $\gamma$ of $\Gamma$ satisfying

$$
|\gamma(t)|=1 \quad \text { and } \quad\langle\gamma(t), B(t) p\rangle \leqq 0
$$

for a.a. $t \in T_{2}$ and all $p \in P(t)$.

By Lusin's theorem there exists a closed subset $T_{3}$ of $T_{2}$ of positive Lebesgue measure such that $\gamma \mid T_{3}$ and the components of $L(\cdot)$ considered as maps on $T_{3}$ with values in the dual of $W^{1,2}\left([-r, 0], \mathbb{R}^{n}\right)$ are continuous. 
Let $\alpha$ be an arbitrary element of $L_{2}\left(T_{3}, \mathbb{R}\right)$ and define $\zeta \in L_{2}\left(T_{1}, \mathbb{R}^{n}\right)$ by

$$
\zeta(t)= \begin{cases}\alpha(t) \gamma(t) & \text { for } t \in T_{3}, \\ 0 & \text { otherwise. }\end{cases}
$$

Define $x^{\alpha} \in W^{1,2}\left(T, \mathbb{R}^{n}\right)$ on $\left[t_{0}, t_{1}-r\right]$ by

$$
x^{\alpha}(t):=0
$$

and on $T_{1}$ as the unique solution of

$$
\dot{x}^{\alpha}(t)=L(t) x_{t}^{\alpha}+\zeta(t) .
$$

Since we assume that $\mathscr{R}=W^{1,2}\left([-r, 0], \mathbb{R}^{n}\right)$, there is an admissible $u^{\alpha}$ such that the corresponding trajectory $y^{\alpha}$ satisfies $y_{t_{1}}^{\alpha}=x_{t_{1}}^{\alpha}$. Hence on $T_{1}$

$$
\dot{x}^{\alpha}(t)=\dot{y}^{\alpha}(t)=L(t) y_{t}^{\alpha}+B(t) u^{\alpha}(t)
$$

and

$$
0=L(t)\left(x_{t}^{\alpha}-y_{t}^{\alpha}\right)+\zeta(t)-B(t) u^{\alpha}(t) .
$$

Taking inner products with $\gamma(t)$ in $\mathbb{R}^{n}$ yields for $t \in T_{3}$

$$
0=\left\langle\gamma(t), L(t)\left(x_{t}^{\alpha}-y_{t}^{\alpha}\right)\right\rangle+\alpha(t)\langle\gamma(t), \gamma(t)\rangle-\left\langle\gamma(t), B(t) u^{\alpha}(t)\right\rangle
$$

or

$$
\alpha(t)=-\left\langle\gamma(t), L(t)\left(x_{t}^{\alpha}-y_{t}^{\alpha}\right)\right\rangle+\left\langle\gamma(t), B(t) u^{\alpha}(t)\right\rangle .
$$

The first term at the right-hand side is continuous, and the second one is negative. This contradicts the choice of $\alpha$ as an arbitrary element in $L_{2}$ and proves (ii).

Remark 2. The assertion of Theorem 2 is not valid for controllability to $W^{1, \infty}\left([-r, 0], \mathbb{R}^{n}\right)$ with $L_{\infty}$-controls. In fact, controllability to $W^{1, \infty}$ only implies that the interior of $B(t) P(t)$ is nonempty for a.a. $t \in T_{1}$. This follows from [3, Thm. 3.3 and Example 3.1], and is a remarkable difference between controllability to $W^{1, \infty}$ and $W^{1,2}$. Taking up the line of argument in [3, Remark 3.3], Theorem 2 shows that one cannot prepare the reaching of an arbitrary $W^{1,2}$-function $\phi$ by a special way of reaching $\phi(-r)$ at $t_{1}-r$. The reason is that in each neighbourhood of $\phi$ there is an element $\xi$ such that $\dot{\phi}-\dot{\xi}$ is unbounded.

Remark 3. In some sense the result of Theorem 2 is negative: Controllability to $W^{1,2}$ can only be achieved if not only the well-known and strong rank condition on $B(t)$ is satisfied, but also the "positivity cone" $P(t)$ is the whole space $\mathbb{R}^{m}$ on the final interval. However, the rank condition is irrelevant for the relaxed optimal control problem (if the control appears nonlinearly). Here $B(t)$ is the identity matrix and generically, int $P(t)=$ int co $f\left(x_{t}^{0}, v^{0}(t)\right) \neq \varnothing$. Furthermore, the condition $P(t)=\mathbb{R}^{m}$ on $T_{1}$ means for the optimal control problem that the control constraint is not active on the final interval. Theorem 2 explains why this strong assumption has to be made.

Proof of Theorem 1. The equivalence of (i) and (ii) is immediate from the proof of Theorem 2. Furthermore, it is clear that (iv) implies (iii). Conversely, suppose that (ii) is violated. Due to our continuity assumption on $\dot{\phi}\left(t-t_{1}\right)=f\left(x_{t}^{0}, v^{0}(t)\right), t \in T_{1}$, this means that there is $\bar{t} \in T_{1}$ with

$$
f\left(x_{\bar{t}}^{0}, v^{0}(\bar{t})\right) \in \partial \operatorname{co} f\left(x_{\bar{t}}^{0}, \Omega\right)
$$

in the linear subspace spanned by $P(\bar{t})$. Thus there exists $\bar{u} \neq 0$ in the linear subspace spanned by $P(\bar{t})$ such that

$$
\langle\bar{u}, p\rangle \leqq 0 \quad \text { for all } p \in P(\bar{t}) .
$$


Then there exists a function $\bar{u}(\cdot)$ in $U_{\infty}\left(T_{1}\right)$ with

$$
\bar{u}(t)=\bar{u} \text { for a.a. } t \text { in a neighbourhood of } \bar{t} \text {. }
$$

However, there is no $\lambda \geqq 0$ such that

$$
\frac{1}{\lambda} \bar{u} \in \operatorname{co} f\left(x_{t}^{0}, \Omega\right)-f\left(x_{t}^{0}, v^{0}(t)\right) \text { a.e. }
$$

This proves the equivalence of (iii) and (iv).

\section{REFERENCES}

[1] C. Castaing And M. Valadier, Convex Analysis and Measurable Multifunctions, Lecture Notes in Mathematics 580, Springer-Verlag, Berlin, 1977.

[2] F. COLONIUS, The maximum principle for relaxed hereditary differential systems with function space end condition, this Journal, 20 (1982), pp. 695-712.

[3] - Stable and regular reachability for relaxed hereditary differential systems, this Journal, 20 (1982), pp. 675-694.

[4] - A note on the existence of Lagrange multipliers, Appl. Math. Optim., 10 (1983), pp. 187-191.

[5] S. Kurcyusz AND A. W. Olbrot, On the closure in $W^{1, q}$ of the attainable subspace of linear time lag systems, J. Differential Equations, 24 (1977), pp. 29-50. 